\title{
Prevalencia de desnutrición infantil en menores de tres años en dos cantones de Ecuador
}

\author{
Mary Consuelo Mosso Ortiz ${ }^{1}$, Mery Rocío Rea Guamán ${ }^{1}$ (D), Katherin Madeley Beltrán Moso ${ }^{1}$, \\ José Ivo Óscar Contreras Briceño ${ }^{2}$ (D),
}

\section{RESUMEN}

Introducción: En Ecuador, de la población de menores de cinco años, uno de cada cuatro niños sufre desnutrición crónica. La situación es más grave para la niñez indígena: uno de cada dos niños la padece: así mismo, cuatro de cada diez presenta algún grado de anemia.

Materiales y métodos: Estudio con orientación cuantitativa, de tipo descriptivo y transversal. La población estuvo conformada por 785 niños de la sierra ecuatoriana, cuyos representantes firmaron un asentimiento informado, previo a la aplicación de la encuesta. Para la estimación de estado nutricional se utilizó el Sistema Informático de Gestión Integral (SGI Nutrición) del Ministerio de Salud Pública.

Resultados: La prevalencia de desnutrición a partir de la relación peso y talla global fue del 18,1\%; la baja talla fue la más frecuente (12,8\%), tomando en cuenta la talla relacionada con la edad. El 29,5\% de los niños presentó algún nivel de desnutrición. La desnutrición según peso y talla tuvo una diferencia importante entre los indígenas (35\%) y los mestizos (17\%).

Conclusiones: La prevalencia de la desnutrición que mide la relación talla y peso (crónica) fue mayor que la de peso para la edad (aguda). Se encontraron diferencias estadísticamente significativas en la desnutrición de acuerdo con la autoidentificación étnica entre mestizos e indígenas.

Palabras clave: estado nutricional; prevalencia; niños; Ecuador.

\footnotetext{
${ }^{1}$ Universidad Estatal de Bolívar, Guaranda, Ecuador.

2 Universidad Católica de Cuenca, Cañar, Ecuador.

${ }^{3}$ Universidad Técnica Particular de Loja, Loja, Ecuador.
}

Autor de correspondencia: José Ivo Óscar Contreras Briceño. Correo electrónico: ivojosebrice@gmail.com

Citar este artículo así:

Mosso Ortiz MC, Rea Guamán MR, Beltrán Moso KM, Contreras Briceño JIO. Prevalencia de desnutrición infantil en menores de tres años en dos cantones de Ecuador. Rev Investig Salud Univ Boyacá. 2021;8(1):18-32. https://doi.org/10.24267/23897325.613 


\title{
Prevalence of Child Malnutrition in Children under Three Years Old in Two Cantons of Ecuador
}

\begin{abstract}
Introduction: In Ecuador, of the population under 5 years of age, the population of 1 in 4 children, aged less than 5 years, suffers from chronic malnutrition. The situation is more serious for indigenous children in which one in two children suffers from it, likewise, four out of every 10 have some degree of anemia.

Materials and methods: A quantitative, descriptive and cross-sectional study. The population was made up of 785 children from the Ecuadorian highlands, whose representatives signed an informed consent, prior to the application of the survey. P, for the estimation of nutritional status, the SGI Nutrition comprehensive management computer system of the Ministry of Public Health was used.
\end{abstract}

Results: The prevalence of malnutrition based on the weight and overall height ratio was $18.1 \%$, being short height was the most frequent $(12.8 \%)$, considering the height related to age, $29.5 \%$ of the children have presented some level of malnutrition; malnutrition according to weight and height, they had an important difference between indigenous (35\%) and mestizos (17\%).

Conclusions: The prevalence of malnutrition that measures the relationship between height and weight (chronic) it was greater than that of weight for age (acute), there are statistically significant differences in malnutrition according to ethnic self-identification between mestizos and indigenous people.

Keywords: nutritional status; prevalence; children; Ecuador. 


\title{
Prevalência da desnutrição infantil em menores de três anos em dois cantões do Equador
}

\begin{abstract}
RESUMO
Introdução: No Equador, da população menor de cinco anos, um de cada quatro crianças sofre desnutrição crônica. A situação é mais severa para crianças indígenas: uma em cada duas crianças sofre com isso: da mesma forma, quatro em cada dez têm algum grau de anemia.

Materiais e métodos: Estudo com orientação quantitativa, de tipo descritivo e transversal. A população esteve constituída por 785 crianças da serra equatoriana, cujos representantes assinaram um consentimento informado, antes da aplicação da pesquisa. Para a estimação da condição nutricional foi utilizado o Sistema Informático de Gestão Integral (SGI Nutrição) do Ministério de Saúde Pública.

Resultados: A prevalência de desnutrição com base na relação peso e estatura global foram de 18,1 $\%$; a baixa estatura foi a mais frequente $(12,8 \%$ ), levando em consideração a estatura em relação com a idade. $029,5 \%$ das crianças apresentou algum nível de desnutrição. A desnutrição segundo o peso e a estatura teve uma importante diferença entre os indígenas (35\%) e os mestiços (17\%).

Conclusões: A prevalência da desnutrição medida pela relação estatura e peso (crônica) foi mais elevada do que o peso por idade (agudo). Diferenças estatisticamente significativas foram identificadas na desnutrição de acordo com a autoidentificação étnica entre mestiços e indígenas.
\end{abstract}

Palavras-chave: condição nutricional; prevalência; crianças; Equador. 


\section{INTRODUCCIÓN}

La desnutrición es el "estado de desequilibrio nutricional, resultado de la insuficiente ingestión de nutrientes para satisfacer los requerimientos fisiológicos normales" (1). Es un problema que repercute de forma sustancial en el crecimiento y desarrollo de millones de niños, porque aumenta los índices de mortalidad y posteriormente, afecta de forma negativa, a aquellos quienes sobreviven a ella. Se evidencia en el retraso del crecimiento de aquellos niños sin acceso a una nutrición adecuada durante los primeros mil días, es decir, desde la concepción hasta los dos años de edad y, con frecuencia, en el resto de la infancia.

A lo largo de su vida, se deben enfrentar a todo lo que representa el retraso en el crecimiento $y$, tal vez, no lleguen a alcanzar completamente el desarrollo personal de sus capacidades, tanto físicas como intelectuales, así como en toda la sociedad $(2,3)$. Según datos de la Comisión Económica para América Latina y el Caribe, hace más de veinte años que esta región se ve afectada por este problema, ya que han encontrado desnutrición en niñas y niños que sufren bajo peso o baja talla durante su primera infancia (4).

En el caso latinoamericano y caribeño, en 2018, uno de cada cinco niños presentaron algún grado de malnutrición, específicamente "4,8 millones de niños menores de 5 años, sufrieron de retraso en el crecimiento y 0.7 millones de emaciación" (2). Otro dato alarmante es que cerca de la mitad de las muertes infantiles tienen la malnutrición como causa subyacente. Aquellos que presentan malnutrición aguda grave son los que poseen una mayor probabilidad de morir a consecuencia de enfermedades, por lo general, que son comunes en los infantes como la diarrea y la neumonía (5).

En Suramérica, se registró por cuarto año consecutivo un aumento de este problema, al sumarse más de 2.000.000 de personas que viven con subalimentación, es decir, que no cubren la ingesta de sus necesidades calóricas que les permita mantener una vida activa y saludable (3).

En este contexto, es importante citar lo descrito por el Fondo de las Naciones Unidas para la Infancia, sobre la malnutrición infantil. El estado nutricional es el resultado inminente de dos causas directas: las enfermedades y la ingesta inadecuada de alimentos (6), toda vez que están definidas por varios elementos causales, a saber: la seguridad alimentaria doméstica, el cuidado de la alimentación adecuada, el ambiente doméstico insalubre y las características de la cobertura universal de salud (7).

Esto significa que para que niñas y niños tengan un adecuado estado nutricional deben tener la disponibilidad física y económica a una variedad de alimentos nutritivos (4), a la atención adecuada 
materna e infantil, a óptimos servicios sanitarios adecuados a la población y a vivir en un entorno saludable que incluya el acceso agua potable, adecuado saneamiento ambiental, así como buenas prácticas de higiene (8).

La desnutrición crónica implica retraso en el crecimiento, es decir, que un niño malnutrido presenta talla baja en relación con su edad. Por su parte, la desnutrición aguda se manifiesta mediante el bajo peso respecto a su altura o bajo peso relacionado con la talla. En Latinoamérica, la forma crónica es un problema que afecta al $67 \%$ de los países y al $10 \%$ de su población $(4,9,10)$. No obstante, existe una gran brecha territorial con relación a la prevalencia de la desnutrición crónica en varios países de la región. Ecuador también presenta estas diferencias.

Un análisis del estado nutricional en Ecuador con la nueva norma de la Organización Mundial de la Salud muestra que la distribución entre los indicadores es ligeramente distinta y diferencial. La desnutrición global se presenta con una prevalencia mayor durante los primeros meses de vida, luego baja, finalmente hace un repunte alrededor de los 15 a 24 meses, que se estabiliza alrededor del valor de los primeros meses de vida o menores (10). Con relación a la desnutrición crónica, en los dos primeros se aprecia un crecimiento importante, que supera al doble a la desnutrición global (11).
Para abril de 2017, en el ámbito nacional, la prevalencia de desnutrición era del 20,1\% de niños (37.416) con baja talla para la edad. En la sierra ecuatoriana, la desnutrición crónica afectaba al $26 \%$ de los niños y niñas, cifra que se eleva drásticamente en el área a rural a un 31,9\% (12), especialmente en las poblaciones indígenas, datos que reflejan las condiciones precarias desde las condiciones económicas y sociales en las que viven los niños que la padecen (12). Para 2018, las provincias del Ecuador con desnutrición que se vieron más afectadas fueron: Bolívar, Santa Elena y Chimborazo, de las cuales Bolívar tiene más niños de 0 a 59 meses, es decir, de cero a cuatro años y nueve meses (13).

En este mismo orden de ideas, en cuanto a la caracterización sociodemográfica de la población objeto de estudio, vale resaltar la eminente pobreza en la provincia de Bolívar y que afecta a un amplio sector de esta población, situación que se recrudece en el ámbito rural, por el limitado acceso a fuentes de trabajo, la precariedad en la oferta de los servicios básicos, así como el acceso limitado a la educación, situaciones que generan un escenario difícil de superar. Todo ello afecta en gran medida a la población femenina, que está inmersa en una sociedad machista y patriarcal que rezaga de manera significativa la posibilidad de tener una vida digna y de potenciar su desarrollo humano y social (14). 
En este sentido, la investigación tuvo como objetivo identificar la prevalencia de desnutrición en niños menores de tres años que asisten a los 48 centros infantiles del Buen Vivir (CIBV) de los cantones Guaranda (46) y Chillanes (2), pertenecientes a la provincia Bolívar (Ecuador).

\section{MATERIALES Y MÉTODOS}

Este fue un estudio con enfoque cuantitativo, descriptivo y transversal. La población estuvo conformada por 785 niños de las edades comprendidas entre 1 y 4 años, 469 hombres $(59,8 \%)$ y 316 mujeres $(40,2 \%)$ que asistían a los CIBV, regentados por el Ministerio de Inclusión Económica y Social en los cantones de Guaranda y Chillanes. Se recolectó la información de la totalidad del universo dada la accesibilidad a esta. Previa firma del asentimiento informado de los padres o representantes legales de la población de estudio, se procedió a preguntar la autoidentificación étnica y a tomar las medidas antropométricas de peso y estatura, basadas en el sistema informático de gestión integral SGI Nutrición del Ministerio de Salud Pública que, a partir de los datos, es un recurso en línea que se accede con la cédula de identidad de cada niño y se agregan las variables antropométricas. Como resultado, el aplicativo emite un diagnóstico del estado nutricional de acuerdo con el peso para la edad y para la talla.
Los datos se analizaron en el Paquete Estadístico para Ciencias Sociales (SPSS, versión 19) y la prueba estadística fue el chi cuadrado $\left(\chi^{2}\right)$ de Pearson en las que se cruzan las variables cualitativas y sus categorías, como puede apreciarse en los cuadros estadísticos en el apartado de "Resultados" y el nivel de significancia con un valor de $p$ menor a 0,05.

\section{RESULTADOS}

En el presente estudio se destaca que el $77,5 \%$ de los niños se encuentra en un rango normal de nutrición; mientras que la prevalencia de la desnutrición a partir de la relación peso y talla global es del $18,1 \%$, en que la baja talla es la más frecuente $(12,8 \%)$, seguido de la baja talla severa $(5,2 \%)$ y la condición de severamente emaciado (0,1\%). Así mismo, el sobrepeso ocurre en el $2,9 \%$ de los niños.

En el primer año de vida es más frecuente la talla baja severa; pero esa tendencia aparece a partir de los dos años y se incrementa hasta los cuatro. Por su parte, el sobrepeso aparece en el primer año y va disminuyendo hasta el final de los cuatro años. Tales aspectos se presentan en la tabla 1. 
Tabla 1. Estado nutricional según peso y edad

\begin{tabular}{lccccc}
\hline \multirow{2}{*}{$\begin{array}{c}\text { Estado nutricional } \\
\text { (peso y edad) }\end{array}$} & \multicolumn{4}{c}{ Edad (años) } & $\mathbf{n}=\mathbf{7 8 5}$ \\
\cline { 2 - 6 } & $\mathbf{1}$ & $\mathbf{2}$ & $\mathbf{3}$ & $\mathbf{4}$ & Total \\
\hline Severamente emaciado & 0,0 & 0,0 & 0,0 & 1,1 & 0,1 \\
\hline Baja talla severa & 4,8 & 6,5 & 5,0 & 4,3 & 5,2 \\
\hline Baja talla & 0,0 & 13,1 & 11,9 & 20,5 & 12,8 \\
\hline Normal & 57,1 & 74,4 & 80,7 & 71,0 & 77,5 \\
\hline Sobrepeso & 19,0 & 4,2 & 2,0 & 2,2 & 2,9 \\
\hline Obesidad & 9,5 & 0,0 & 0,0 & 0,0 & 0,3 \\
\hline Talla alta & 9,6 & 1,8 & 0,4 & 1,1 & 1,0 \\
\hline Total & 100,0 & 100,0 & 100,0 & 100,0 & 100,0 \\
\hline
\end{tabular}

En la tabla 2 está reflejado el estado nutricional tomando en cuenta la talla relacionada con la edad. La prevalencia de esta relación pondoestatural fuera de los valores normales es del $29,5 \%$, es decir, 3 de cada 10 niños tiene algún grado de desnutrición (al agregar severamente emaciados, emaciados, baja talla y baja talla severa). En este sentido, la baja talla representa $19 \%$ y la baja talla severa está en el 9,9\% del total de la población, que implica es más de tres veces mayor en el primer año que en el resto de los años. En cuanto a la baja talla, es vista en uno de cada cinco niños $(19 \%)$ y se distribuye de manera más o menos igual por cada año de vida. La proporción de los infantes en el rango normal disminuye al $66 \%$; la baja talla aparece de nuevo como la principal categoría de desnutrición, y luego la baja talla severa, con el 9,9\%. Las condiciones de emaciado o severamente emaciado evidencian muy poca frecuencia. Por el lado de la nutrición por exceso, es más frecuente el sobrepeso con el 3,6\%.

Tabla 2. Estado nutricional de acuerdo con la talla y la edad

\begin{tabular}{lccccc}
\hline \multirow{2}{*}{$\begin{array}{c}\text { Estado nutricional } \\
\text { (talla y edad) }\end{array}$} & \multicolumn{4}{c}{ Edad en años } & \multicolumn{3}{c}{$\mathbf{n = 7 8 5}$} \\
\cline { 2 - 6 } & $\mathbf{1}$ & $\mathbf{2}$ & $\mathbf{3}$ & $\mathbf{4}$ & Total \\
\hline Severamente emaciado & 4,8 & 0,6 & 0,4 & 0,0 & 0,5 \\
\hline Emaciado & 0,0 & 0,6 & 0,0 & 0,0 & 0,1 \\
\hline Baja talla severa & 33,3 & 8,9 & 9,9 & 6,5 & 9,9 \\
\hline Baja talla & 14,3 & 19,6 & 18,7 & 20,4 & 19,0 \\
\hline Normal & 42,9 & 64,3 & 66,4 & 72,0 & 66,0 \\
\hline Obesidad & 4,8 & 0,0 & 1,0 & 0,0 & 0,8 \\
\hline Sobrepeso & 0,0 & 5,4 & 3,6 & 1,1 & 3,6 \\
\hline Talla alta & 0,0 & 0,6 & 0,0 & 0,0 & 0,1 \\
\hline Total & 100,0 & 100,0 & 100,0 & 100,0 & 100,0 \\
\hline
\end{tabular}

Como se aprecia en la figura 1, se establece la relación entre las variables del peso y de la talla. De manera general, el $78 \%$ se encuentra en estado nutricional normal, aun cuando ello es ligeramente menor en los niños mestizos (76\%), es decir, la desnutrición es del $17 \%$ entre los indígenas y del $20 \%$ en mestizos (baja talla y baja talla severa, emaciados y severamente emaciados). Entre los problemas más frecuentes de nutrición se tiene: la baja talla ( $17 \%$ en mestizos y $11 \%$ en indígenas) y la baja talla severa. 
Figura 1. Estado nutricional según la relación peso-talla y autoidentificación étnica

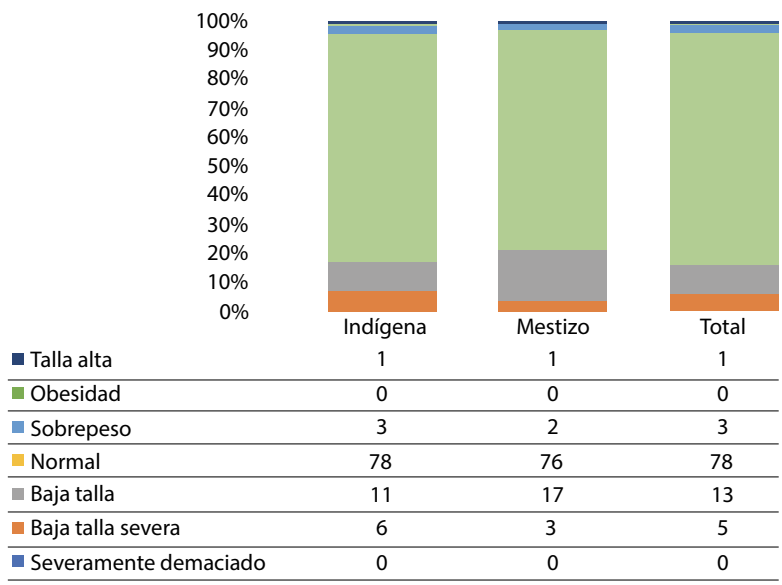

La figura 2 muestra la relación de la edad y la talla (estatura). Se puede notar que la desnutrición tiene una diferencia importante entre los indígenas ( $35 \%)$ y los mestizos (17\%). Adicionalmente, la baja talla para la edad es mayor entre los indígenas ( $21 \%)$; igualmente, la baja talla severa $(13 \%)$.

En la tabla 3 se establecen las relaciones entre las variables demográficas (edad y autoidentificación étnica) con el estado nutricional (peso-edad considerada para medir la desnutrición aguda y peso-talla para la crónica). En todas las mediciones se encontraron diferencias estadísticamente significativas a un nivel de significancia del $95 \%$ o valor de $p$ menor a 0,05.
Figura 2. Estado nutricional según la relación talla-edad y autoidentificación étnica

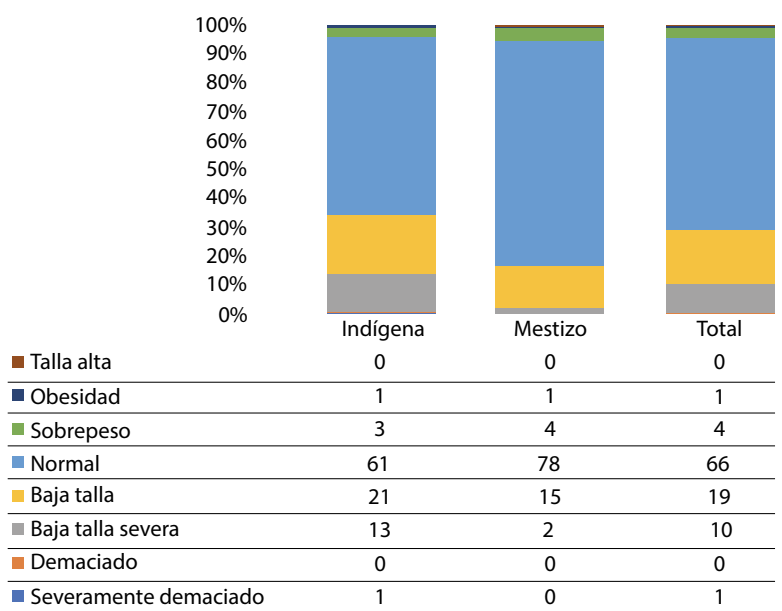

Tabla 3. Chi cuadrado (Pearson) de variables demográficas con el estado nutricional según peso-edad y peso-talla

\begin{tabular}{lcc}
\hline Cruce de variables & $\begin{array}{c}\text { Chi cuadrado } \\
\text { de Pearson }\end{array}$ & Valor de $\boldsymbol{p}$ \\
\hline $\begin{array}{l}\text { Estado nutricional (peso y edad) } \\
\text { versus edad (años) }\end{array}$ & 202,8 & 0,102 \\
\hline
\end{tabular}

Estado nutricional (peso y talla) versus edad (años)

19,7

0,238

Estado nutricional (peso y edad) versus autoidentificación étnica 


\section{DISCUSIÓN}

Las etapas o variantes de la desnutrición van desde la emaciación (peso bajo con respecto a la talla), el retraso del crecimiento (una talla insuficiente para la edad) y la insuficiencia ponderal (un peso insuficiente para la edad) (15).

En esta investigación, la emaciación es prácticamente inexistente; sin embargo, está presente el retraso del crecimiento en uno de cada seis niños (18\%) e insuficiencia ponderal en tres de cada de diez niños (28,9\%). Un estudio realizado en Ecuador reporta desnutrición con cifras similares a este estudio $(8,9 \%)(16)$; mientras que en otro se reportan cifras mayores (17). Así mismo, otra investigación reportó que el $21,9 \%$ de los niños tenía un retardo leve de baja talla para la edad, y el 3,6\%, un retardo moderado o grave de baja talla para la edad, y solo un $10 \%$ de retraso de peso para la talla (18). Por otro lado, en Carchi se encontraron resultados más altos de desnutrición crónica (25,7\%) (19); mientras que en la parroquia de Pifo se reporta una prevalencia de desnutrición crónica del 15,8\%, y una aguda del $2,0 \%(20)$.

En términos comparativos con investigaciones nacionales, la prevalencia de desnutrición aguda y crónica encontrada en este estudio es superlativamente mayor; además. supera el promedio de la zona andina ecuatoriana (25,4\%) (21). Entre otras causas que influyen en el fenómeno en estudio, es importante destacar la evidente pobreza que vive la población que reside en el contexto geográfico del estudio, que puede incidir determinantemente en las cifras encontradas (14).

Respecto a las tendencias nacionales, esta investigación revela cifras inferiores. En ese sentido, la desnutrición infantil afecta a uno de cada cuatro de la población de 0 a 4 años. Ecuador, como un país de ingreso medio, tiene un alto grado de malnutrición infantil (22).

En el ámbito internacional, se encontró en Honduras mayor prevalencia de emaciación (10\%), y el $53 \%$ tenía un adecuado estado nutricional (23). En una investigación en Colombia se encontró desnutrición crónica en el mismo grupo etario $(35,5 \%)$, con predominio en la población indígena (24)especialmente en comunidades indígenas. Objetivo: Determinar la prevalencia de parasitosis intestinal, anemia y desnutrición en niños de un resguardo indígena Nasa de Caldono, en el Departamento del Cauca, y su distribución según variables clínicas, sociodemográficas y de infraestructura sanitaria. Métodos: Estudio transversal con fuente de información primaria. La muestra de estudio estuvo formada por 62 niños a quienes se les hicieron evaluación parasitológica en materia fecal, mediciones antropométricas para evaluar el estado nutricional y determinar la prevalencia de diferentes tipos de desnutrición, medición de hemoglobina para establecer la anemia. 
La descripción del grupo se realizó con medidas de resumen para la edad y frecuencias para las demás variables, se calculó la prevalencia de los tres eventos (parasitosis, desnutrición, anemia; mientras que en el mismo país un trabajo evidenció una malnutrición por déficit, del 9,8\% (25). Por otra parte, en Perú una investigación muestra una prevalencia de desnutrición crónica del $44,87 \%$ y un $2,56 \%$ de desnutrición aguda (26).

Respecto a los países cercanos citados, las diferencias pueden estar influenciadas por el acceso a servicios, que se determina según el grado de desarrollo en detrimento del área rural. En una visión global, puede decirse que la desnutrición es un reflejo de las condiciones económicas, sociales y ambientales en las que vive el niño.

En este sentido, los niños con atraso en el crecimiento, relacionado con determinantes como una alimentación impropia o infecciones recurrentes, tienden a tener un mayor riesgo de enfermedad y muerte. Por su parte, la demora en el crecimiento se debe a una carencia de nutrientes durante años $y$, a menudo, da como resultado un retraso en el desarrollo mental, un rendimiento escolar deficiente y una capacidad intelectual reducida. Estos resultados impactan en la productividad económica nacional, más allá de sus repercusiones en la salud pública. Las mujeres de baja estatura tienen un mayor riesgo de complicaciones obstétricas, debido a una pelvis más pequeña.
Las mujeres pequeñas, como es el caso de las ecuatorianas, tienen mayor riesgo de engendrar un bebé con bajo peso al nacer, lo que contribuye a la perpetuación de la desnutrición en generaciones posteriores (27). La alimentación, conjuntamente con otros determinantes, tiene un rol fundamental para el desarrollo intelectual, físico y social de los escolares, y ello repercute en el resto de sus ciclos vitales (28).

Según lo anterior, la nutrición infantil tiene características que se alejan de las prácticas saludables, mientras que la lactancia materna antes de los 6 meses de vida muestra diferencias entre los ámbitos rural $(70,1 \%)$ y urbano $(58,4 \%)$. Ello también se manifiesta para la desnutrición crónica en menores de 5 años, en el área urbana (20,1\%) y rural (28,7\%), así como el sobrepeso y obesidad en niños de 5 a 11 años en el espacio urbano $(36,9 \%)$ y rural $(32,6 \%)$. Como puede apreciarse, los indicadores muestran dos realidades diferenciadas por áreas poblacionales $(29,30)$.

Entre los hábitos alimentarios del país, se estima que alrededor del $65,2 \%$ de los niños y niñas de 6 a 23 meses de edad ingieren alimentos fortificados o ricos en hierro, y que el quintil más pobre es el que tiene el menor consumo $(59,4 \%)(31)$.

De acuerdo con documentos oficiales, el país tiene un rezago importante en combatir la desnutrición crónica, evidenciado por desigualdad 
social y los problemas económicos que afectan con mayor intensidad a la población pobre. Ello complica duramente el potencial de desarrollo de las personas y el conglomerado social. Entre los factores que inciden en la desnutrición están: acceso insuficiente a alimentos de calidad; inadecuadas prácticas de alimentación y cuidado; enfermedades infecciosas; falta de agua, vivienda y saneamiento deficiente, y dificultades para el acceso sanitario universal (32-34).

En el mismo orden de ideas, otros factores que generan desnutrición, debido a que exacerban la pérdida de peso, son el parasitismo y las diarreas persistentes, dado que el $29,9 \%$ de la población no tiene acceso a agua segura, cercana, suficiente y libre de contaminación con Escherichia coli. El $14 \%$ no tiene servicios higiénicos adecuados en el hogar; además de que el $12,7 \%$ no tiene instalaciones para el lavado de manos dentro de la vivienda, con mayor fuerza en zonas rurales (35).

En este punto de la discusión, aún persisten retos para lograr los Objetivos de Desarrollo Sostenible por parte del Estado ecuatoriano, según la agenda 2015-2030 de las Naciones Unidas. En este orden de ideas, eliminar la pobreza, poner fin al hambre, lograr la seguridad alimentaria y la mejora de la nutrición y eliminar la desnutrición crónica requieren el despliegue de grandes e importantes esfuerzos para lograr su cumplimiento (34).

\section{CONCLUSIONES}

La prevalencia de desnutrición global, medida por la relación peso y talla, es cercana a uno en cada cinco niños de la población. La baja talla para la edad es la condición más común y en una proporción mucho menor se encuentra la baja talla severa. Las cifras indican que en niños de un año la talla baja severa es la primera condición; sin embargo, la tendencia cambia en los años sucesivos.

Cerca de uno de cada tres niños tiene alguna condición de desnutrición valorada, y al relacionar la talla con la edad, la baja talla es el más frecuente. Al asociar el estado nutricional con la autoidentificación étnica, la desnutrición según pesos y talla es similar; ligeramente mayor entre los mestizos, y en las formas más frecuentes, como lo son la talla baja y su versión severa.

Esta misma relación, pero con la medición de la talla y edad, permite observar una diferencia importante entre los indígenas, que es uno de cada tres niños, y en los mestizos es casi la mitad que en la población indígena. También la baja talla para la edad es mayor entre los indígenas

\section{AGRADECIMIENTOS}

A la Universidad Estatal de Bolívar (Ecuador). 


\section{FINANCIAMIENTO}

La investigación es autofinanciada por los autores.

\section{CONFLICTO DE INTERESES}

Los autores declaran no tener conflicto de intereses.

\section{DECLARACIÓN DE CONTRIBUCIÓN}

Todos los integrantes participaron de manera equitativa en el proceso investigativo en su integralidad, la recolección de la información científica actualizada; además de la redacción del artículo.

\section{REFERENCIAS}

1. Organización Mundial de la Salud, Organización Panamericana de la Salud. Desnutrición: descriptores en ciencias de la salud. Washington; 2020.

2. Fondo de las Naciones Unidas para la Infancia. El estado mundial de la infancia 2019 en América Latina y el Caribe: niños, alimentación y nutrición. Crecer bien en un mundo en transformación [internet]. Asunción: Unicef; 2019. Disponible en: https://www.unicef.org/ lac/informes/el-estado-mundial-de-la-infancia-2019-ni \%C3 \%B1 os-alimentos-y-nutrici \%C3\%B3n
3. Organización de las Naciones Unidas para la Alimentación y la Agricultura (FAO), Organización Panamericana de la Salud (OPS), Programa Mundial de Alimentos (WFP), Fondo de las Naciones Unidas para la Infancia (Unicef). Hacia el logro del hambre cero y una vida sana para todas las personas. En: Panorama de la seguridad alimentaria y nutricional en América Latina y el Caribe: desigualdad y sistemas alimentarios. Santiago: FAO; 2018. p. 19-29.

4. Naciones Unidas, Comisión Económica para América Latina. Malnutrición en niños y niñas en América Latina y el Caribe. Washington; 2018.

5. Organización Mundial de la Salud. Reducción de la mortalidad en la niñez. Geneva; 2019.

6. Fondo de las Naciones Unidas para la Infancia. La mala alimentación perjudica la salud de los niños en todo el mundo [Internet]. 2019 oct 15 [citado 2021 jul 25]. Disponible en: https://www.unicef.org/es/comunicados-prensa/la-mala-alimentación-perjudica-la-salud-de-los-niños-en-todo-el-mundo-advierte

7. Fondo de las Naciones Unidas para la Infancia (unicef) Venezuela. Mejorar la nutrición infantil: el imperativo para el progreso mundial que es posible lograr. Caracas; 2013. 
8. Organización de las Naciones Unidas para la Alimentación y la Agricultura (FAO), Organización Panamericana de la Salud (OPS), Programa Mundial de Alimentos (WFP), Fondo de las Naciones Unidas para la Infancia (Unicef). Dimensiones de la seguridad alimentaria y nutricional. En: Panorama de la seguridad alimentaria y nutricional en América Latina y el Caribe: desigualdad y sistemas alimentarios. Santiago: FAO; 2018. p. 43-8.

9. Fondo de las Naciones Unidas para la Infancia (Unicef). 5 formas de acabar con la desnutrición infantil [internet]. 2021. Disponible en: https://www.unicef.es/blog/5-formas-de-acabar-con-la-desnutricion-infantil

10. Martínez R, Palma A. Seguridad alimentaria y nutricional en cuatro países andinos: una propuesta de seguimiento y análisis [internet]. Santiago de Chile: Naciones Unidas; 2014. Disponible en: https://repositorio.cepal.org/bitstream/ handle/11362/36773/1/S20131121_es.pdf

11. Fernández A, Martínez R, Carrasco I, Palma A. Impacto social y económico de la malnutrición: modelo de análisis y estudio piloto en Chile, Ecuador y México. Santiago: Naciones Unidas; 2017.

12. Ministerio de Salud Pública del Ecuador. Plan Intersectorial de Alimentación y Nutrición, 2018-2025. Quito; 2018.
13. La erradicación de la desnutrición infantil, otra deuda de Ecuador. El Comercio [internet]. 2018 ene 22. Disponible en: https://www.elcomercio.com/pages/ninos-ecuatorianos-registran-baja-estatura.html

14. Sagredo J, Galarza A. Economía rural e inequidad de género en el cantón Chillanes, provincia de Bolívar. Rev Investig Enlace Univ. 2020;19(1):49-62. https://doi.org/10.33789/ enlace.19.1.59

15. Organización Mundial de la Salud. Malnutrición. Washington; 2020.

16. Guanga V, Carpio T, Moreno M, Silva S. Desnutrición aguda infantil en la Zona 3: estudio ecológico-descriptivo del "Sistema de Seguimiento de Salud del MSP" del Ecuador. 2016-2018. Cienc Serv Salud [internet]. 2020;11(Esp):3141. Disponible en: http://revistas.espoch.edu. ec/index.php/cssn/article/view/496

17. Blacio Vidal WJ. Anemia y estado nutricional en menores de 5 años: Hospital Pablo Jaramillo Crespo, Cuenca-Ecuador. Rev Fac Cienc Méd Univ Cuenca. 2020;38(3):47-55. https://doi. org/10.18537/RFCM.38.03.05

18. Chiriboga Calva SDR, Guamaní Heredia DJ. Factores determinantes de la desnutrición crónica en los niños menores de 5 años de la Frontera 
Norte del Ecuador [internet]. Quito: UCE; 2020 [citado 2021 jul 25]. Disponible en: http:// www.dspace.uce.edu.ec/handle/25000/21868

19. Pérez Ponce JL. Prevalencia de desnutrición en niños menores de 5 años en el Centro de Salud San Antonio-lbarra 2020 [internet]. Ibarra: Universidad Técnica del Norte; 2021 [citado 2021 jul 25]. Disponible en: http://repositorio. utn.edu.ec/handle/123456789/11023

20. Guamialamá Martínez J, Salazar Duque D, Portugal Morejón C, Lala Gualotuña K. Estado nutricional de niños menores de cinco años en la parroquia de Pifo. Nutr Clín Diet Hosp. 2020;40(2):90-9. https://doi.org/10.12873/ 402guamialama

21. Ramos-Padilla P, Carpio-Arias T, Delgado-López V, Villavicencio-Barriga V. Estado nutricional antropométrico de niños menores de 5 años de la región interandina del Ecuador. Rev Esp Nutr Comun. 2020;26(4).

22. Rivera J. La malnutrición infantil en el Ecuador. RevEstud PolítPúblicas. 2018;5(5):1-108. https:// doi.org/10.5354/0719-6296.2019.51170

23. Delcid Morazán AF, Delcid Morazán LE, Barcan Batchvaroff ME, Leiva Molina FA, Barahona Andrade DS. Estado nutricional en escolares de primero a sexto grado en la Paz, Honduras. Rev Cient Esc Univ Cienc Salud. 2019;4(1):2733. https://doi.org/10.5377/rceucs.v4i1.7066

24. Gaviria LM, Soscue D, Campo Polanco LF, Cardona Arias JA, Galván Díaz AL. Prevalencia de parasitosis intestinal, anemia y desnutrición en niños de un resguardo indígena Nasa, Cauca-Colombia, 2015. Rev Fac Nac Salud Pública. 2017;35(3):390-9. https://doi.org/10.17533/ udea.rfnsp.v35n3a09

25. Barrera D, Fierro E, Puentes L, Castañeda J. Prevalencia y determinantes sociales de malnutrición en menores de 5 años afiliados al Sistema de Selección de Beneficiarios para Programas Sociales (SISBEN) del área urbana del municipio de Palermo en Colombia, 2017. Univ Salud. 2018;20(3):10. https://doi. org/10.22267/rus.182003.126

26. Oroya Surichaqui J. Prevalencia de desnutrición en menores de 5 años en el Distrito de Huacrapuquio, 2018 [tesis de grado en internet]. Huancayo: Universidad Peruana Los Andes; 2019 [citado 2021 jul 25]. Disponible en: http://repositorio.upla.edu.pe/ handle/20.500.12848/553

27. World Health Organization. Nutrition Landscape Information System (NLIS) country profile indicators: interpretation guide. Washington; 2010. 
28. Núñez RB, Zambrano MQ, Alarcón MS, Monar LV, Cisneros JC. Alimentación saludable como factor influyente en el rendimiento escolar de los estudiantes de instituciones educativas en Ecuador. Facsalud-Unemi. 2017;1(1):34-9. https://doi.org/10.29076/issn.2602-8360voI1 iss1.2017pp34-39p

29. Instituto Nacional de Estadística y Censos. Salud, salud reproductiva y nutrición. Encuesta Nacional de Salud y Nutrición-ENSANUT 2018 [internet]. 2020. Disponible en: https:// www.ecuadorencifras.gob.ec/salud-salud-reproductiva-y-nutricion/

30. Arias P, Neira J. Desnutrición escolar. [Latacunga]: Universidad Técnica de Latacunga; 2017.

31. Freire WB, Ramírez-Luzuriaga MJ, Belmont $P$, Mendieta MJ, Silva Jaramillo K, Romero N, et al. Encuesta Nacional de Salud y Nutrición de la Población Ecuatoriana de cero a 59 años. Tomo 1. [internet]. Quito: Ministerio de Salud Pública-Instituto Nacional de Estadísticas y Censos; 2014 [citado 2017 sep 27]. Disponible en: http://www.ecuadorencifras.gob.ed/ documentos/web-inec/Estadisticas_Sociales/ ENSANUT/MSP_ENSANUT-ECU_06-10-2014.pdf

32. Kline $L$, Jones-Smith J, Jaime Miranda J, Pratt M, Reis RS, Rivera JA, et al. A research agenda to guide progress on childhood obesity prevention in Latin America. Obes Rev. 2017;18(S2):19-27. https://doi.org/10.1111/ obr.12572

33. Observatorio Social del Ecuador. Situación de la niñez y adolescencia en Ecuador: una mirada a través de los ODS. Quito; 2019.

34. Velasco M, Álvarez S, Carrera G, Vásconez A. La niñez y adolescencia en el Ecuador contemporáneo: avances y brechas en el ejercicio de derechos [internet]. Quito: Observatorio Social del Ecuador; 2014. Disponible en: https:// biblio.flacsoandes.edu.ec/catalog/resGet. php? resld $=53951$

35.Instituto Nacional de Estadísticas y Censos (INEC). Indicadores ODS Agua, Saneamiento e Higiene. Quito: Instituto Nacional de Estadísticas y Censos (INEC); 2016.

Esta obra está bajo una licencia de Creative Commons Reconocimiento-NoComercial 4.0 Internacional 